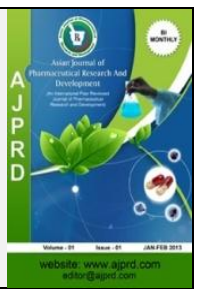

Open $\odot$ Access

Review Article

\title{
A Review on Pharmacovigilance Process In India
}

\author{
Preti Mishra $^{1 *}$, Pawan Upadhyay ${ }^{2}$, Ravi Rawat $^{2}$, Tajamul Ashraf dar $^{2}$, Kapil Dev ${ }^{2}$, Nidhi Chauhan ${ }^{2}$ \\ ${ }^{1}$ Raja Balwant Singh Engineering Technical Campus, Bichpuri - Agra, U.P., India \\ ${ }^{2}$ Arya college of Pharmacy, Kookas, Jaipur, Rajasthan, India
}

\begin{abstract}
A B S T R A C T
Peoples are using more potent drugs with various medical conditions. pharmacovigilance helps in safe and convenient use of pharmaceutical drugs. Voluntary recording of adverse drug reactions (ADRs) is a chief component of pharmacovigilance. Adverse drug reactions have become a dominant health related problems in developing countries like India. The main objective of pharmacovigilance is the assessment of benefit-risk profile of drug for better potency and safety in patients. In terms of volume India pharmaceutical industries is third largest in the world so India has a core of clinical research and drug design \& development. This review article explains the need of pharmacovigilance in pharma companies, the growth of pharmacovigilance in different centuries and current status of pharmacovigilance in the country.
\end{abstract}

Key words: Pharmacovigilance, Thalidomide Disaster, anti-inflammatory drugs.

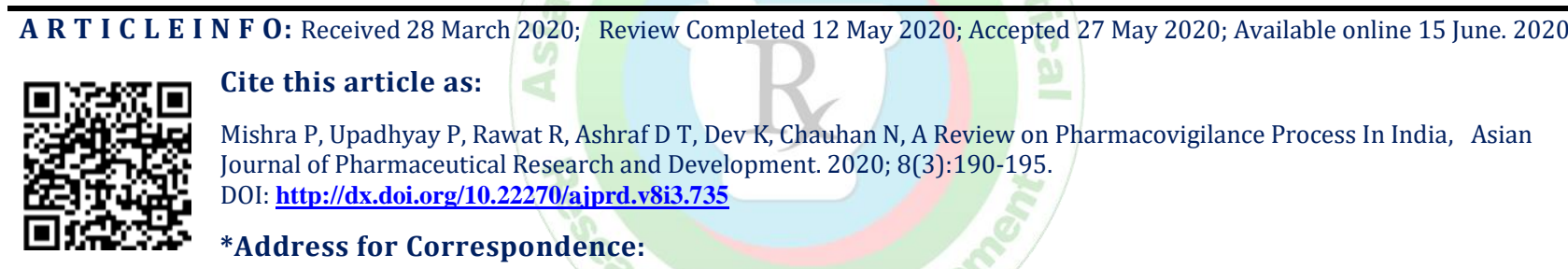

Preti Mishra, Raja Balwant Singh Engineering Technical Campus, Bichpuri -Agra, India.

\section{INTRODUCTION}

A dverse drug reactions (ADRs) are the dominant reason of fatality in the world. Pharmacovigilance Programme of India (PvPI) is prepared as supervising authority by Indian Pharmacopoeia Commission (IPC) in favour to protect the community health systems. Pharmacovigilance is the system of compilation, supervising, analysis, estimation and interpretation of data provided from patients and medical professionals on injurious drug reactions or other drug related complications. Pharmacovigilance deals with indivisual adverse drug reactions or medication errors caused by Pharma products ${ }^{1}$. The aim of the Pharmacovigilance is analyzing the different information about medication risk ${ }^{2-3}$. The Pharmacovigilance programs assures the safety of drugs and promote the systematic and rational uses of drugs. It develops the public health, patient responsibility and their safety ${ }^{4-5}$. There are different fields which encourages Pharmacovigilance programs such as Pharmaceutical industries, medical writing, pharmacists, regulatory affairs and clinical practices ${ }^{6-8}$. Dying from illness is consistently obligatory; dying from medicine is undesirable ${ }^{9}$. Pharmacovigilance focused on drug surveillance programs and its process involves:

- Compile and report of AEs/ADRs.

- Causality assessment and study of ADRs.

- Collect and combine the database.

- Calculate risk-benefit ratio and support regulative action.

- Convey for secure use of drugs between participants.

History of pharmacovigilance:

\section{Pharmacovigilance before the $18^{\text {th }}$ Century}

On reviewing the literature before the $18^{\text {th }}$ century, it reveals the evidences of people alertness concerning the probabilities of noxious and therapeutic effects of drugs attempted for regenerate various diseases. Few such examples are mentioned here. In $1780 \mathrm{BC}$ the Babylonian Code of Hammurabi gives abuses for damage done by therapeutic treatments. According to this explanation: "If a specialist form a huge incision with the surgical knife and kill him or open a humor with the surgical knife and cut out 
the eye, his hands shall be cut off". In the 10th century Salerno Medical School was permitted to inspect medicines for possible contamination and strict fine were enforced. "However if someone buy or sell any poisonous drug not needed to his profession, let him be swing". In the 13th century the pledge of Apothecaries in Basle, Switzerland, mentions the uses of drugs by physicians. Medicines should be of such good nature and of such convenience that he knows, upon bis oath, that it will be great and beneficial for the confection what the practitioner is created. In $1599 \mathrm{BC}$, King James VI of Scotland expressed a document to make concession for the guidance of sales of drugs and poison. William Spang was the first auditor appointed and he shared the authority of acceptance of drugs for selling in Glasgow. All above mention evidences from literature clearly indicate the keen interest of man in taking steps to eliminate the adverse effects combined with therapeutic effects, However the amount taken were localized to the small group of people and organized efforts to involve wider population did not exist.

\section{Pharmacovigilance in the $18^{\text {th }}$ Century}

In the 18th century, People established more attempts to compromise with safety related issues and Drugs having severe adverse drug reactions were recorded by health care professionals. There are certain examples of the adverse reactions of drugs during the 18th century; some are discussed here. An English physician William Withering (1741-1799) disclosed his comprehensive task on digitalis in 1785. This task was later accepted as the first efficient article on drug remedies with the comprehensive explanation of adverse reactions combined with foxglove treatment.

In his book entitled "A report of the foxglove and part of its Medicinal Uses: With Practical assertion in edema and other Diseases gives a detailed account of methods for the digitalis synthesis from Foxglove in a standardized manner, details of biological effects and symptoms combined with digitalis overdoses. In the agreement with this explanation, Foxglove when administered in huge and several doses starts a regurgitation, expulsion, vertigo, visual disturbance, enhance urine flow, increased the frequency of motion, slow pulse, cold, sweating, convulsions, insensibility and death. He emphasized on the need appropriate evaluation of doses. Today the awareness of therapeutic activities of digitalis has increased significantly but the basic symptomatology associated with overdoses still remains the same. The Royal College of Physicians commemorates the achievements of William Withering in an annual lecture named after him. In 1789, Wouter van Doeveren, Professor of Medicine (Fig. 2.2) at Leiden University and a critic of the medical practice of that time, discussed in his academic session named Remedio Morbi, the disorders which generally influence peoples as a result of the administration of drugs for curative purposes. In his lecture van Doeveren mentioned that physicians often consider the treatment related problems as either essential or hazardous and are seldom moderate in their judgment. He also pointed out the hazards associated with commonly used therapeutic methods at that time such as bloodletting and perspirationinducing drugs for acute fever and concluded that many illnesses may result from improper treatments carried without proper diagnosis. He warned that a second disease may be added to the first or maybe the death results as a consequence of experimental treatment. This lecture indicates the emerging scientific activities in injurious drug reactions in the 18 th century.

In the 18th century Calomel (mercurous chloride) was extensively utilize in America for the treatment of the onset of yellow fever. Treatment starts the mercurialism characterized by extensive salivation, loosening the teeth, lesions, gangrene and osteomyelitis of the mandible. Eventually the adverse reactions physicians continued to use Calomel. Later Oliver Wendell Holnics in 1861 said, "If whole Materia Medica as it is so used would be sure to the basement of the sea it would be all superior for mankind and all the bad for the fishes".

The 18th century review literature exhibits the alertness of the peoples mainly the scholars and specialists, who started Communication by different ways such as lectures and publications, related to the safe use of drugs.

\section{Pharmacovigilance in the $19^{\text {th }}$ Century}

The reports of organized recording of adverse drug reactions correlated with beneficial parts are applicable from the 19th century. One such example of organized reporting involving cowpox vaccine is described here. The cowpox vaccination initiated from 1718 and indeed of demand provided related its potency and security, the cowpox vaccination was not commonly approved. A positive response was only observed after the publication of the work of Edward Jenner on variolac vaccines in 1798. With the help of Royal College specialists a campaign was dispatched in the Netherlands for cowpox inoculation in 1808 with the attention of the compilation of data related to the effects of Cowpox vaccine and another particularity that may be observed. The process not only proved the benefits of the vaccine but also demonstrate safety. Systematic methods were also followed to evaluate ADRs related to chloroform anesthesia.

In 1848 death of 15 years old girl observed by given chloroform anesthesia resulting ingrown toenail was recorded. In 1880, The British Medical Association to inquiry organized Glasgow Committee due to the higher rate of death by chloroform anesthesia. The committee resulted that "Chloroform was noxious to the heart and more harmful than other medications".

Later in 1888 in Hyderabad, Edward Lawrie declared safe use of chloroform in 40000 peoples

without any mortality. As a result 'First Hyderabad Chloroform Commission' was selected to verify the claim and after conducting experiments in 141 animals the commission resulted that chloroform may be securely used for anesthesia if a respiration is attentively examined. That report was not authorized in England and the 'Second Hyderabad Chloroform Commission' was assembled for reinvestigation. The second commission also included a representative from Lancet. Then again study was carried out in 430 animals and 54 humans. The second commission approved the findings of the Glasgow Committee.

In a similar development in 1848 a statute was passed in America to regulate the nature of medicaments when quinine transported for the US army was found adulterated. In 1881, a book published by L Lewin regarding organized reporting of ailments combined with pharmacotherapy programs. 


\section{Pharmacovigilance in the $20^{\text {th }}$ Century: Prethalidomide Era}

During 20th century evidences showed to the higher authorities for control of drug synthesis. The FDA was organized in 1906 to enforce condition for manufacturing of drugs and Pharmacopoeias. 1938 was the memorial year when security demand was started and constituted related to sulfanilamide disaster. In 1937, sulfa compounds were treated as 'wonder drugs' they destroy a broad area of destructive bacteria. In 1938, manufacturer formulated an elixir of sulfanilamide by diffusing the medicament in diethylene glycol. After that, 107 people including 100 children were dead by using sulfanilamide elixir, prepared in a harmful solvent (diethylene glycol).

In June 1938, the Federal Food, Drug and Cosmetic Act were registered in the USA. This act enforced the security assurance of new medicaments before come in the market and it was necessary that new drug application (NDA) integrates the security information. This act also needed proper labelling of drugs for safe use. In 1950, the same response carried through safety purpose with the blood dyscrasia registry by the council of the American Medical Association. 9,10

An overview of the available literature on pharmacovigilance was published by L Meyler in Dutch in 1951. After 1952, the English translation entitled "Side Effects of Drugs" was also declared and subsequently translation was done in other languages. ${ }^{11,12}$ Later a number of publications followed and one such publication in 1955 by HL Alexander was entitled "Reactions includes Drug Therapy". Also in 1955 an article published by Barr in JAMA reported that $5 \%$ of the patients admitted to the hospital suffer from major toxic reactions and accidents. A number of reports published later also mentioned similar figures. ${ }^{13,14}$

\section{Pharmacovigilance in the $20^{\text {th }}$ Century: Thalidomide Disaster and Post-Thalidomide Era}

The thalidomide, which was first manufactured in 1953 was extensively encourage in 1956 under several brand names in Germany, England and other countries and was accessible as prescription and over-the-counter drug. An Australian specialist McBride first analyzed an interaction among maternal need of thalidomide and congenital malformation in newborns. In June $1961 \mathrm{He}$ consigned a document to Lancet in this concern, which got declared in December 1961 in a pediatric conference congenital malformation with parenteral usage of thalidomide. As this affiliation of thalidomide with congenital disorder got more and more consideration, the producer Chemie Grunenthal withdraw thalidomide from the market on 25th November 1961. Later it was reported that nearly 6000-12000 children had inherited disorder because of parental need of thalidomide and majority of them were born in Germany. 14,15

Thalidomide tragedy led to present some severe measures in many countries. The FDA started an organized compilation of records on all types of injurious drug reactions. In another countries along with potency the safety of medication was indicated and definitives were confirmed in which the new drugs were needed to accomodate before pursuing authority for retailing, Government organizations were advised to set up a phase IV clinical trial methods so as to take up the adverse drug reactions as soon as available and prevent similar tragedies in the future. In 1968, the ten countries in the world contribute with the WHO for an International drug safety surveillance program. As the result of enhancing alertness to improve safe use of drugs various adverse drug reactions started and noticed by physicians. In 1969, an extensive digitalis related disaster was disclosed in the Dutch town of Veenendaal. The Physician Dr. AH Lely discovered the development of serious symptoms of digitalis toxicity over a period of about 2-3 months. Subsequently, it was revealed that due to production error the distributed tablets were composed of digitoxin $0.20 \mathrm{mg}$ and digoxin 0.05 instead of recommended digoxin $0.25 \mathrm{mg}$. At least 19 deaths were attributed to this production. One particular aspect of this act is to notify the capability of the specialist to analyze the symptoms relevant to overdose and inform them to others. It is also noticeable that other physicians who also must have observed that similar symptoms, failed to bring them to the notice of others either because they were not aware of the symptoms or simply did not inform others. Knowledge, keen observation and efficient reporting still form the basics of Pharmacovigilance. ${ }^{15,16}$

\section{Pharmacovigilance: The Current Status}

In 20th World Health Assembly, Authority of the World Health Organization (WHO) International Drug Monitoring Program was established in 1971. The recent international system of pharmacovigilance is depending on the paper disclosed in 1972 and appropriately the national pharmacovigilance centers were settled that effort in participation with the WHO. ${ }^{17}$

A WHO Association Center for International Drug Monitoring is depends on Uppsala, Sweden. (The Uppsala Surveillance Center, supports and coordinates the WHO International Drug Monitoring Program). The WHO center provides an effective support to the pharmacovigilance centers in developing states. This center collected the pharmacovigilance data from national pharmacovigilance centers, maintain the international database, assess the ability and issues of ongoing national pharmacovigilance programs and takes measures to more build up them with professional and economic support. In 2000, WHO Uppsala Surveillance Center supplied instructions for working a pharmacovigilance center. In 2002, WHO publication the concern of Pharmacovigilance prepared the instructions for fulfillment of the pharmacovigilance schedule at international level. The achievement of progressing, implementing the new constitution and qualitative requirements get to the formation of the Council of International Organizations of Medical Sciences (CIOMS) and the International Conference on Harmonization (ICH). These institutions along with national regulatory authorities and pharmaceutical industries have been involved in the progress of Pharmacovigilance world-wide.

\section{Pharmacovigilance: The Current System in India}

A formal drug safety monitoring system was suggested for the first time in India in 1986. An adverse drug reaction observation consist of 12 local centers and particular center covered a 50 million population. More detailed efforts of drug safety monitoring in India started in 1997, in partnership with WHO Uppasala Monitoring Center. Under this program three adverse drug reaction monitoring centers were recognized including a National Pharma- covigilance Center at All India Institute of Medical Sciences (AIIMS), 
New Delhi and two WHO special centers at Mumbai and Aligarh. This program did not accomplished due to several reasons leading to launch of a more ambitious National Pharmacovigilance program (NPP) guarantee by WHO and finance by the World bank on 1st January, 2005. The aim of NPP is to engage a huge number of medical experts in this case, impart the art of describing adverse drug reaction and for standards overall drug observation. ${ }^{18,19}$

\section{Need For Pharmacovigilance}

Today, the need for an efficient Pharmacovigilance system has been realized more than ever to ensure safe use of drugs. There are multiple reasons for this increasing necessity for pharmacovigilance and some of these includes in Box 3.1. Unreliability of preclinical safety data when the drug is marketed for the first time the experiences with concern of safety and potency are mainly depends on the conclusion of clinical trials. As the clinical trials are usually managed under well-controlled situation, it is very tedious to conclude potency, adverse effects and the total riskbenefit ratio under the certain clinical setting. During the drug development process research mainly focused on evaluation of the efficacy of drugs. Although the adverse effects are also determined but their value in real life situations is limited. The preclinical drug improvement process involves the assessment of drug safety and the efficacy in animal experiments and generally it may not be convenient to conclude the results of animal experiment to human. The clinical trials involving human volunteers are usually done under strictly controlled conditions involving a small sample size (rarely the sample size is more than 3000). The data collected is selective and confidential. The clinical trials generally do not associate with special groups of people (children, elderly, pregnant) and are not done under the conditions usually encountered in clinical practice. This made difficult to anticipate the occurrence of adverse drug effects in the special group of peoples and in special cases such as co administration with other drugs or in disease conditions. ${ }^{20,21}$

The premarketing safety evaluation of drugs often may not be as reliable as expected because of

pressure from patient groups, pharmaceutical industry management, political groups and regulatory authorities to reduce the time taken for approval. As a time for approval decreased the possibilities of detecting unexpected adverse drug reactions also decreased.

\section{The Priority Areas of Pharmacovigilance}

The regulation of Pharmacovigilance has advanced considerably since the 1972. Presently, the pharmacovigilance priority fields that desired to be convey at national and international level have been composed by the WHO and involve the following risk of ADRs:

- It develops the exact analysis of adverse drug reactions by healthcare workers and patients. Strengthen the effective examination of individual drug safety involve over epidemiological methods such as case control studies and epidemiological survey. It examined the appropriate activities needed for the analysis of security related to vaccines, biological, veterinary medicines, herbal medicines, biotechnological products and investigational drugs. It improved marked exposure arrangement by promoting the presence of information that may have international importance. It promote and appliance the
ADR analyzing systems that may favorable to populations with the restricted approach to medical management and judgment of ADRs. ${ }^{22}$

- Another advancement of automatic signal exposure method utilize in voluntary surveillance program.

- Advancement in judgment of medicine security that have universal importance. ${ }^{23}$

- Encourage collective channels at the local and international level that could confess countries to check and behave accordingly to medicine security disasters. It examined the approaches by which knowledge on the arrangement of drug usage can be incorporated with Pharmacovigilance data at the time of judgment of welfare and impairment at a national level avoidance of ADRs.

- Improves approaches to predictable and impartial information at different health care centers.

- Improves approaches to safe and potent medications for ignoring the disease frequent in established societies. ${ }^{24}$

- Integrate pharmacovigilance activities into national drug usage between health care practitioners and the public incorporate pharmacovigilance programs into national drug approaches and the action begin from these typical analysis instructions and essential drugs etc. Then again fusion of Pharmacovigilance assumptions into analytic uses and intellectual medicines inspired the assumptions of a product administered in the peoples of health care. It improves the supervision of conventional and herbal medicines. ${ }^{25,26}$

In response to drug security complications, Pharmacovigilance system determined the preventive action taken against adverse drug reactions. It improves participation between colleagues in Pharmacovigilance both locally and internationally. The fundamentals of the good communication process in pharmacovigilance are drug control should be determined and the system expertise to convey was accepted. ${ }^{27,28}$ It improves understanding of patients, their predictions of drugs and approaches of exposure related with the usage of medications ${ }^{29}$. It appreciates the programs that will suggest the public related to benefit and misuse of medicines. It develops secure and efficient link in order to promote efficient and specific drug knowledge to the peoples. It established the compatibility between drug regulation and Pharmacovigilance programs by incorporate the wide international company in the advancement of affiliated approaches. Impact and result manage the ongoing investigation to determine the charge efficiency of current pharmacovigilance systems in the commitment to the patient health benefit. It examined the awareness and particularity of present signal disclosure and estimation approaches and the degree to which new pharmacovigilance systems have been passed in analyzing and defending possible hazards although escaping the immature elimination of secure and convenient drugs from the market. ${ }^{30}$

\section{The Outcome and Impact of Current System}

The national and international Pharmacovigilance programs studied the probable serious adverse drug reactions and that decision has been taken in the past to remove the probable harmful drugs from the market. One such example of the drug withdrawal from the market is described here. The 
VIOXX Saga Non steroidal anti-inflammatory drugs (NSAID) like aspirin and ibuprofen act primarily by inhibiting enzymes cyclooxygenase 1 and 2 (COX 1 and COX 2) thereby, it inhibits the prostaglandin synthesis. COX 1 is a constitutively present enzyme which is required for a variety of body functions such as protection of gastric mucosa whereas COX 2 plays an essential role in inflammation. With the identification of two separate COX enzymes and their functions it was realized that any drug that can selectively inhibit COX 2 will be devoid of adverse effects so commonly associated with nonselective COX inhibitors like aspirin. Hence, the birth of Rofecoxib, popularly known as "Vioxx', a selective COX 2 inhibitor, which at its inception held the promise of pain relief in rheumatoid arthritis, acute pain and dysmenorrhea without the dreaded adverse effects of nonselective COX inhibitors. [31]Major human clinical trial including a systematic evaluation of more than 8000 patients demonstrated that Vioxx kept therapeutic efficacy without gastric toxicity. Considering that more than 16,500 people in the US died from NSAID related gastrointestinal bleeding, this discovery was of great significance and as a result all the shareholders of Merck \& Co. reaped the benefits. However, on September 30, 2004 Merck \& Co. declared spontaneous removal of Vioxx depends on new data from a clinical trial 'Adenomatous polyp prevention on Vioxx' (APPROVe). It was a rigorous,randomized, placebo-controlled doubleblind study involving 2600 patients for over 3 years. The trial was executed to estimate the efficiency of Vioxx 25 mg inhibiting the frequency of colorectal polyps in patients with colorectal adenomas. The investigation was terminated prematurely when an increase in the exposure of cardiovascular diseases like myocardial infarction and cardiac stroke was noted. The important fact to notice from this clinical trial is that results from the first 18 months did not exhibit an increase in exposure of cardiovascular cases in Vioxx treated patients as compared to placebo. ${ }^{32,33}$

\section{CONCLUSION}

The drug security surveillance is an important component for the safe usage of drugs and for good quality medicinal responsibility. It has the probability to motivate assurance and faith between patients and health care practitioner in drugs and committed to raise parameters of medical system. Besides, the adverse drug reactions significantly diminish the quality of life, increase the rate and duration of hospitalization. Thus, it increased the mortality and morbidity. The financial burden on health care authorities increases enormously. As the newer discoveries are becoming available to the needy population at a faster rate due to several recent trends in approval and regulations, the drug-related adverse reactions are also becoming very common, severe and complex. Clearly, the formulation and fulfillment of a highly efficient Pharmacovigilance program, which can meet the required objectives is of prime importance at national and international levels.

\section{REFERENCES}

1. Pirmohamed M, James S, Meakin S, Green C, Scott AK, Walley TJ et al. Adverse drug reactions as cause of admission to hospital: prospective analysis of 18820 patients. BMJ. 2004 Jul 1; 329(7456):15-9.

2. Patel TK, Patel BP. Incidence of adverse drug reactions in Indian hospitals: a systematic review of prospective studies. Curr Drug Saf. 2016 Apr 1;11(2):128-36.

3. Mcbride WG. Thalidomide and congenital abnormalities. Lancet. 1961; 278(7216):1358.
4. Sharma G, Kumar R, Singh J, Bhandari V, Singh N. Pharmacovigilance in India and it's impact in patient management. J Curr Trends Diagnosis Treatment. 2017; 1(1):27-33.

5. Thota P, Thota A, Medhi B, Sidhu S, Kumar P, Selvan VK, et al. Drug safety alerts of pharmacovigilance programme of India: a scope for targeted spontaneous reporting in India. Perspectives Clin Res. 2018 ; 9(1):51.

6. Kalaiselvan V, Thota P, Singh GN. Pharmacovigilance Programme of India: Recent developments and future perspectives. Indian J Pharmacol. 2016; 48(6):624.

7. Kalaivani M, Kalaiselvan V, Dabhi K, Singh GN. Direct consumer reporting of adverse drug reactions to PvPI, a position paper of IPC. Adv Pharmacoepidemiol Drug Saf. 2015; 4(3):184.

8. Pharmacovigilance programme of India, 2010. Available at: www.who.umc.org/pharmacovigilance.html. Accessed 14 May 2018.

9. Indian Pharmacopoeia Commission Newsletter PvPI. 2015;5(12):4 Available at: www.ipc.gov.in/PvPI/newsletter/Newsletter\%20Vol $\% 205 \% 20$ Issue $\% 2012 \% 202015 \% 20$ PDF.pdf

10. Singh KNM, Kanase HR. Pharmacovigilance Programme of India: the beginning, current status and recent progress. Adv Pharmacoepidemiol Drug Saf. 2017; 6(4):219.

11. Indian Pharmacopoeia Commission Newsletter PvPI. 2015; 5(11):68. Available at: www.ipc.gov.in/PvPI/newsletter/Newsletter\%20Vol $\% 205 \% 20$ Issue\%2011\%202015\%20PDF.pdf Accessed 14 May 2018.

12. Pharmacovigilance guidelines for marketing authorization holders of pharmaceutical products in India, 2018. Available at: www.ipc.gov.in. Accessed 14 May 2018.

13. Pharmacovigilance programme of India, 2010. Available at: http://www.ipc.gov.in/PvPI/adr.html. Accessed 14 May 2018.

14. Power outage at Puducherry hospital kills three dialysis patients The Hindu dated 9 March 2017. Available at: http://www.thehindu.com. Accessed 25

15. 15.WHO Policy Perspectives on Medicines. Geneva: WHO; 2004. Geneva: World Health Organization. Looking at the Pharmacovigilance: ensuring the safe use of medicines.

16. Klepper MJ. The periodic safety updates report as a Pharmacovigilance tool. Drug Saf 2004; 27:569- 78. 3. Livio F, Renard D, Buclin T. Pharmacovigilance. Rev Med Suisse 2012; 8:116-9

17. 17.WHO, Safety of medicines in public health programmes: Pharmacovigilance an essential tool,WHO,2006.

18. Skalli S, Soulaymani Bencheikh R. Safety monitoring of herb-drug interactions: acomponent of pharmacovigilance. Drug Saf 2012; 35:785-91.

19. Arnott J, Hesselgreaves H, Nunn AJ, Peak M, Pirmohamed M, Smyth RL, et al. What can we learn from parents about enhancing participation in pharmacovigilance?. Br J Clin Pharmacol 2012; in press.

20. 20.WHO, Pharmacovigilance: ensuring the safe use of medicines, Geneva: WHO 2004.

21. WHO, Safety of medicines in public health programmes: pharmacovigilance an essential tool,WHO,2006.

22. WHO Medicines Strategy: Framework for Action in Essential Drugs and Medicines Policy 2000- 2003. 23. Olsson S. The role of the WHO Programme for International Drug Monitoring in coordinating worldwide drug safety efforts. Drug Saf 1998; 19:110.

23. WHO, Pharmacovigilance: ensuring the safe use of medicines, Geneva: WHO 2004.

24. ICH Guideline E2D; Post-approval safety data management: definitions and standards for expedited reporting, 3.1.1 Spontaneous reports 2004. International Conference on Harmonisation.

25. Pinkston V, Swain EJ. Management of adverse drug reactions and adverse event data through collection, storage, and retrieval. In: Stephens MDB, Talbot JCC, Routledge PA, eds. Detection of new adverse drug reactions. 4th ed. London, MacMillan Reference Ltd, 
1998:282. http://z.umn.edu/INNOVATIONS 2015, Vol. 6, No. 1, Article 189

26. Faich GA, U.S. adverse drug reaction surveillance 1989-1994 Pharmacoepidemiology Drug Safety, 1996, 393-398. 15. Goldman SA. Limitations and strengths of spontaneous reports data. Clinical Therapeutics, 1998, 20 (Suppl C):C40-C44.

27. Hartmann K, Doser AK, Kuhn M. Postmarketing safety information: How useful are spontaneous reports. Pharmacoepidemiology and Drug Safety, 1999, 8:S65-S71.

28. Waller PC, Arlett PA. Responding to signals. In: Mann RD Andrews E, eds. Pharmacovigilance. Chichester, John Wiley and Sons Ltd, 2002:105-128.

29. DuMouchel W. Bayesian data mining in large frequency tables, with an application to the FDA Spontaneous Reporting system. The American Statistician, 1999; 53:177-190.
30. Bate A, Lindquist M, Edwards IR. A Bayesian neural network method for adverse drug reaction signal generation. European Journal of Clinical Pharmacology, 1998; 54:315-321.

31. Van Puijenbroek E, Egberts ACG, Heerdink ER, Leufkens HGM. Detecting drug-drug interactions using a database for spontaneous adverse drug reactions: An example with diuretics and nonsteroidal anti-inflammatory drugs. European Journal of Clinical Pharmacology, 2000; 56:733-738.

32. Venning GR. Identification of adverse reactions to new drugs. III: Alerting processes and early warning systems. British Medical Journal, 1983; 286:458-460.

33. Edwards IR. The management of adverse drug reactions: from diagnosis to signal. Thérapie, 2001; 56:727-733. 